To understand the human genome, researchers must spread their wings to all branches of life.

$\wedge$ $s$ the technology for gene sequencing becomes more powerful, the prospect of personalized medicine draws ever closer. On 4 September, genomics pioneer Craig Venter followed in the footsteps of James Watson and published his complete genome sequence (see page 6). Plans are already being laid for hundreds more personal genomes to be sequenced (see Nature 447, 358-359; 2007).

But a publication that will attract rather less attention than these personal genomes illustrates why researchers should ensure that genomic data are collected not just from humans and their closest relatives, but from every far-flung branch of the tree of life.

The paper in question focuses on segments of 'ultraconserved' DNA - sections that have stayed exactly the same throughout recent vertebrate evolution, and are identical in humans, rats and mice (see page 10). The available evidence suggests that this extreme example of DNA conservation is no accident: the sequence stays because there is a strong selective force weeding out mutations in it. In other words, it is likely to be important to its host.

Yet when researchers based at Lawrence Berkeley National Laboratory in California removed four pieces of ultraconserved DNA from different mice, it had absolutely no effect on the rodents (N. Ahituv et al. PLoS Biol. 5, e234; 2007). This counterintuitive result contradicts predictions based on genetic conservation and the shaping of our genomes during evolution. Reconciling it with what scientists currently know would be easier if geneticists could figure out where ultraconserved DNA comes from, or what its function might be. Researchers have suggested that it could be involved in splicing RNA transcripts (J. Z. Ni et al. Genes Dev. 21, 708-718; 2007) or in enhancing transcription (L. A. Pennacchio et al. Nature 444, 499-502; 2006).

But so far there has been just one report on the origin of some ultraconserved DNA (G. Bejerano et al. Nature 441, 87-90; 2006). A team from the University of California, Santa Cruz, traced the origins of one ultraconserved region back to a group of ancient fishes, including the coelacanth. This was only possible because another group of researchers had previously opted to sequence a few segments of coelacanth DNA. As Gill Bejerano, a former member of the Santa
Cruz team, says: "If the coelacanth people hadn't been interested in that puny $1 \%$ of the genome we would not have the answer. Who knows what other information is out there?"

It is clear that efforts to understand the mechanisms of evolution will benefit from getting as much genetic information on as many diverse organisms as possible. As it happens, the US National Human Genome Research Institute (NHGRI) announced in May that it would add a fly and a worm to its ENCODE project, which aims to catalogue all the functional parts of the human genome, in order to meet that project's human goals (see Nature 447 , 361; 2007).

The NHGRI and the other main public backer of genomics research in the United States, the Department of Energy, are each committed to com-
"A better understanding of DNA function will come only from generating data from diverse genomes." parative genomics. But under the influence of the 'roadmap' of the US National Institutes of Health (NIH), which emphasizes the translation of research findings into the clinic, the NHGRI is moving more forcefully into purely human genomics. The biggest new projects recently announced by the NHGRI are all human-centric, such as the Cancer Genome Atlas and a pair of initiatives to hunt for the genetic causes of human disease - the Genetic Association Information Network and the Genes, Environment and Health Initiative. The institute would also like to embark on a major human cohort study (F. S. Collins and T. A. Manolio Nature 445, 259; 2007), and it is setting up a strategy for sequencing the genomes of our closest relatives, the non-human primates.

This is all understandable enough: the public is entitled to expect that the results of NIH research will be useful, when possible, to public health. But a better understanding of DNA function and the consequence of mutation will come only from generating more data from diverse genomes, backed by the bioinformatics capability that is needed to annotate them. That way scientists can learn more about the extent of DNA conservation throughout the living world - and, ultimately, tease out a deeper comprehension of the human genome.

\section{The big splash}

\section{An unforeseeable chain of insights into an event 65 million years ago merits celebration.}

W hen it comes to sensational science, the story of the asteroid impact some 65 million years ago at the boundary of the Cretaceous and Tertiary periods is hard to beat. The event itself must have been spectacular, with a vast fiery hole blown in Earth's crust and atmosphere, and tsunamis racing out from the impact point, kilometres tall. Its legacy, too, was impressive, dealing an apparent coup de grâce to a group of animals, the dinosaurs, that had dominated the continents for more than 100 million years. The science that brought this extraordinary event to light came with drama of its own - a startling announcement from a team that included a high-profile Nobel prizewinning physicist, Luis Alvarez, followed by decades of sometimes acrimonious debate as asteroid proponents and volcano supporters battled like titans.

The science of the $\mathrm{K} / \mathrm{T}$ impact ( $\mathrm{K}$ is the customary abbreviation for Cretaceous) began in a more modest way, with attempts to get a sense of how quickly a thin layer of clays in the Italian Apennines had 
been deposited. No one foresaw that it would change how scientists and others see the world, and reintroduce catastrophism to the Earth sciences. Explanations that ignore the once-canonical principles of uniformitarianism - the gradualist paradigm in which the present is the key to the past - are now rife in studies of the history of Earth. A sense of Earth's abiding connection to the cosmos beyond is now more widely felt in the scientific community.

More broadly, the idea that Earth might be subject to such insult again at some time in the future has become a topic for box-office blockbusters and for sensible research and policy-making. The various surveys that were set in motion in the 1990s have reduced by almost an order of magnitude the risk of collision with a previously undiscovered asteroid that could drastically affect the global environment.

Against the background of these sweeping ideas, it is good to pause and take stock of the fact that there is still room for detail — indeed, paper on the K/T impact was published in 1980 (L. W. Alvarez et al. Science 208, 1095-1108; 1980). Geologists are now seriously proposing that they might be able to date the events of 65 million years for details that would have been scarcely imaginable when the first

ago to within 25,000 years - which is to say by better than 1 part in 2,500 (see page 20). Meanwhile, astronomers are saying with about $90 \%$ confidence that the asteroid that struck Earth on that darkest of days was a sibling of the asteroid Baptistina. Both seem to have been chips off a larger block that was destroyed 160 million years ago in a catastrophe of its own (see pages 30 and 48).

When the idea of an impact was first mooted, the notion of fleshing
"It is good to pause and take stock of the fact that there is still room for detail." out the story to this degree of elaboration - with the impact site characterized, the timing set to extraordinary accuracy, and even the source of the utterly destroyed impactor narrowed down to a particular parent — was all but unthinkable.

The ability of diverse sciences to collectively provide a coherent story about a time so distant is the sort of triumph that enthusiasts for interdisciplinary approaches should single out for praise and emulation. They should also celebrate the scientific pursuit of ever finer detail. After all, this Earth-shattering narrative began with just such attention to a centimetre-thin stratum.

The outlook for the tigers at Hengdaohezi is also coloured by officials at the State Forestry Administration and elsewhere saying that China plans to reopen trade in tiger body parts for use in traditional Chinese medicine. Indeed, farming the tigers for this use was the initial purpose of the Hengdaohezi centre when the government opened it in 1986. It is only thanks to a ban enacted in 1993 in compliance with the Convention on International Trade in Endangered Species of Wild Fauna and Flora that the facility switched to tourism and conservation research.

It is easy, of course, for outsiders to criticize such practices without acknowledging that nations have more things to think about than animal conservation. And some observers have voiced support for the proposed trade in tiger parts, saying that it could allow local people to make a living, while potentially relieving poaching pressure on the wild tiger population.

But most conservationists have condemned any repeal of the ban on the tiger trade. Even if, as proponents suggest, a legal trade did reduce the price of body parts, prices would remain high enough to encourage poaching. Conferring legal status on the trade would, in the end, increase trading volumes and put more pressure on the wild population.

At the Hengdaohezi conservation facility, meanwhile, poor breeding
"Giving legal status to the trade in tiger body parts would put more pressure on the wild population." wild ahead of next year's Beijing Olympics. If this plan went ahead would almost certainly fail, exposing animals that have grown accustomed to human attention to the mercy of their wild counterparts, possibly with fatal results.

The pattern at the Hengdaohezi facility is, unfortunately, typical of animal conservation efforts in China. Despite recent improvements in its breeding strategy, the Wolong Giant Panda Research Center in Sichuan province, for example, has not been sufficiently open about incidents such as the death of Xiang Xiang, a panda released into the wild last year. management and detachment from the international conservation community has produced a group of docile animals that would stand little chance of survival in the wild.

Breeding and conservation are two very different things. If China is serious about conserving Siberian tigers, or other endangered species, it should concentrate more on the conservation of natural habitats, together with the careful monitoring of animal health, and less on the rapid breeding of animals in confinement. 